event, because the event disproportionately affects the nanotube properties and the nanotube has such a large optical cross-section. Other groups are exploring different technologies to detect single-molecule interactions.

Taekjip Ha is a physicist from the University of Illinois at Urbana-Champaign who has been developing fluorescence resonance energy transfer (FRET) approaches for studying single-molecule interactions. "It took several years to convince ourselves that this technique was going to be very useful to obtain biophysical and biological measurements," says $\mathrm{Ha}$.

Although some studies using single-molecule FRET have been done in living cells, the vast majority of experiments have been in vitro. "This is due to technical limitations," says Ha, "the probes are not bright enough for robust measurements in living cells." But he does think that the use of quantum dots could enable the more widespread use of single-molecule FRET in living cells - with one small change. "It is currently difficult to do a single-molecule
FRET experiment using quantum dots because those made commercially are too big."

A quantum dot plus its coating, which is needed to solubilize the dot and conjugate to a biomolecule, can easily be more than 20 nanometres in diameter - much larger than the 10 nanometres maximum separation required for a FRET experiment. Ha thinks that single-molecule FRET in living cells will have to wait until the next generation of quantum dots. He thinks this is only a matter of time, as he sees no reason why quantum dots cannot be made smaller.

\section{Setting the trap}

"The area of single-molecule manipulation goes back to 1991," recalls Bustamante. "At that time we did an experiment using gravity and the weight of little beads to extend the DNA." Bustamante and others soon turned their attention from beads and gravity to a more sophisticated technique called 'optical tweezers', developed by Stephen Chu, currently at University of California, Berkeley, and Arthur Ashkin, now retired from Bell Labs and Lucent Technologies. This technique can trap and manipulate molecules with diameters of nanometres.

Optical tweezers make use of a focused laser beam to trap and hold dielectric objects. Developed in the late 1980s and used extensively in the 1990s, researchers only recently started to see a need for even higher-resolution optical tweezer systems. "I think during that earlier time our objective was to be able to follow and extend molecular motors, see molecules unfold and measure the mechanical properties of nucleic acids," says Bustamante, "and you could do quite a bit of research without going into extremely high resolution." But today, Bustamante thinks the biology has actually pulled biophysicists towards studying things at a smaller scale - requiring more stability, less drift, more spatial resolution and improved temporal resolution from optical tweezers.

For high-resolution optical tweezers to work, the key issue of drift had to be overcome. One approach, developed by Steven Block's group

\title{
WHEN ONE CANTILEVER IS NOT ENOUGH
}

"I did my doctoral work building force and optical microscopy systems," says Daniel Fletcher, a bioengineer at the University of California, Berkeley. "I was an instrument developer and did not really get to use them, as the main goal was always to develop a new contrast mechanism or achieve the best resolution." But all that changed when Fletcher started his postdoc training and saw cells crawling.

"I realized that although we could build these fancy instruments that control forces down to the piconewton level or below, we had no clue how to assemble complex molecular systems that do what the crawling cells could do." This led Fletcher to study the dynamic actin networks of crawling cells using atomic force microscopy (AFM) and optical traps.

The biochemistry of the actin system has been studied for more than 20 years and actin networks can be grown in vitro, which provides the opportunity to directly probe how network assembly is involved in force generation and shape-changes in cells. "What we have been trying to do with both AFM and optical traps is to create a controlled resistance to

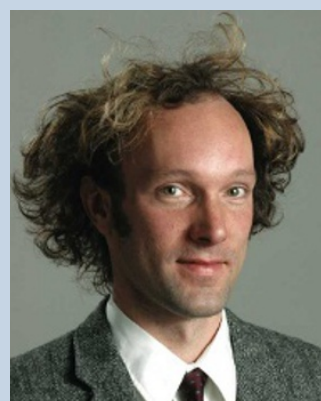

Daniel Fletcher studies actin networks using atomic force microscopy. the growth of these networks and study their behaviour," he says. One approach that Fletcher's group uses is to grow actin networks on the ends of its AFM cantilever and then monitor how the networks adapt to different loads and how those forces influence the architecture of the structures generated.

Using AFM in this manner provided a challenge, requiring Fletcher to use his instrumentdevelopment skills. "One of the things that AFM is not good at doing is measuring sustained displacement over long time periods, because the surface can drift relative to the cantilever,"

\section{says Fletcher.}

Cantilevers are not directly connected to the surface being scanned - they are held by a fluid cell, held by a mount, which is connected to a moving stage that is connected through an even longer path to the surface. The distance between cantilever and surface helps to make crucial calculations of the extent of deflection of the cantilever by the surface. Fletcher explains that because of this large mechanical path from the surface to the cantilever, which can alter the reference position of the cantilever relative to the surface due to thermal drift, the forces measured over time can change even if the sample does not. At short time scales this might not be an issue, but for his team, trying to measure actin growth continuously on the microscope tip over hours, drift of this sort can potentially influence the data collected.

Fletcher decided that one approach to correcting for this drift would be a dual cantilever
AFM system. Having two adjacent cantilevers allows one cantilever to always be in contact with the surface while the second measures the growth of the actin networks. Fletcher says that by always having one cantilever on the surface, they can now detect movement or drift directly and use feedback to correct the cantilever's position.

Fletcher continues to develop new approaches to making force measurements. His team recently developed an epi-fluorescence 'side-view' AFM device (most commercial systems come with the AFM system mounted on top of a microscope) to visualize growing actin networks between the cantilever and the surface. "Where people interested in single-, or even multimolecule, biophysics can continue to make contributions is in developing better tools that help to overcome the limitations of existing technologies and reveal new behaviour of biological systems."

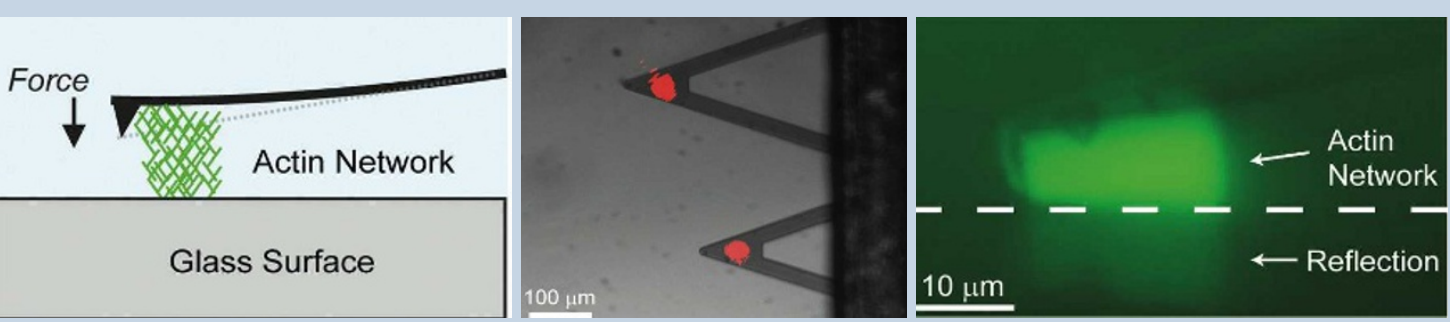

Side-view AFM (left) and the two-cantilever approach (centre) for studying actin-network growth. 